\title{
Electrical Properties of Carbon Nanotubes Cement Composites for Monitoring Stress Conditions in Concrete Structures
}

\author{
Luigi COPPOLA $^{1 \mathrm{a}}$, Alessandra BUOSO ${ }^{1 \mathrm{~b}}$, Fabio CORAZZA ${ }^{2 \mathrm{c}}$ \\ ${ }^{1}$ Faculty of Engineering, University of Bergamo, Italy \\ ${ }^{2} \mathrm{CTG}$ - Italcementi Group - Bergamo, Italy

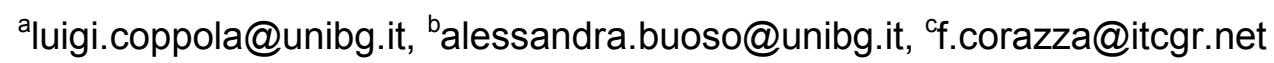

Keywords: Cement, Carbon nanotubes (CNTs), Pressure-Sensitivity.

\begin{abstract}
Cement pastes reinforced with Multi-Walled carbon NanoTubes (MWNTs) are smart materials with piezoresistivity properties. Adding carbon nanotubes to the cement matrix, in fact, the electrical resistivity of cementitious composites changes with the stress conditions under static and dynamic loads. This particular behaviour can be used to evaluate the stress level in reinforced concrete structures, to monitor the traffic flow, to weigh vehicles. In this paper data on pressuresensitive behaviour under compressive stress of cement pastes and mortars containing different percentages (from $0.0 \%$ to $1.0 \%$ vs. cement mass) of MWNTs are presented.In order to form a conductive network and enhancethe piezoresistive properties of cementitious mixtures, Carbon NanoTubes (CNTs) need to be efficiently dispersed in the cement matrix. Two different methods to disperse CNTsin the cement matrix were used. The first one uses a surfactant (Sodium Linear Alkyl Benzene Sulphonate - LAS): MWNTs were dispersed in a LAS aqueous solution, and thenmixed with cement and a defoamer (tributyl phosphate) to decrease the air bubble in MWNT filled cement-based composites. The second method consists in mixing CNTs with about $50 \%$ of the mixing water in a becker by means of a glass wand. Then, the solution is sonicated by an ultrasonic generator for 10 minutes. Finally, the sonicatedCNT-aqueous solution ismixed with cement (and sand for the mortars). The piezoresistivity properties of the cementitious mixtures manufactured with the two above mentioned CNTs dispersing methods will be compared.Experimental results show that the electrical resistance changes synchronously with the compressive stress levelsfor the specimens manufactured with both methods. Therefore, CNTs improve the pressure-sensitivity of cementitious composites. Moreover, the piezoresistive response is better for cementitious composites manufactured by using the surfactant agent to disperse CNTs. Data indicate that thanks to the better dispersion of nanotubes promoted by the surfactant - the pressure-sensitivity properties of cement pastes can be achieved even by using a very low percentage of CNTS $(0.1 \%$ vs. cement mass). These findings seem to indicate that self-sensing CNTs/cement composite can be produced. These smart materials have great potential and they could be used in the next future in concrete field for practical applications to monitor the stress level of reinforced concrete elements subjected to static, dynamic and impact loads. In particular, informations on actual stress existing under dynamic and impact loads could be improve design procedures in protective structures.
\end{abstract}

\section{Introduction}

In 1991, Sumiolijima [1] used a high resolution transmission electron microscope (TEM) in order to study the particulate created in an electric discharge between two carbon electrodes. He discovered that this particulate contained structures consisting of a lot concentric microtubules of graphitic carbon with outer diameters of $4-30 \mathrm{~nm}$ and a length of up to1 $\mu \mathrm{m}$, the carbon nanotubes.CNTs are formed from a graphite sheet (that is hexagonal networks of carbon atoms) rolled up to pipe form, with the two closed extremities by hemispherical caps.Single Wall carbon NanoTubes (SWNT) are constituted by only one graphite sheet and Multi Wall carbon NanoTubes (MWNT) are formed by sheets positioned as concentric cylinders inserted one inside the other. The diameter of a SWNT ranges from $0.4 \mathrm{~nm}$ to $10 \mathrm{~nm}$ and their length is usually of the micrometer order. The diameter of the MWNTs is greater than the SWNTs, reaching tens of nanometers [2]. 
CNTs have a high tensile strength up to about 100 times than steel, despite their specific mass is six times lower [2]. CNTs possess exceptional electrical and thermal conductivity [3]. Therefore addition of carbon nanotubes in electrical insulating polymeric or cementitious matrix can change dramatically electrical conductivity. It was evidenced that the electrical conductivity of polymer would increase about 108 times when carbon nanotubes were added in the amount of 7-11\% (by polymer mass). In the same way addition of carbon nanotubes in cementitious mixtures determines a decrease in the electrical resistivity. Moreover electrical resistivity of cementitious mixtures containing carbon nanotubes changes with stress level in the composite [4]. Variation in the electric resistivity can be ascribed to the increase in the number of contact points of CNTs by enhancing loading: the higher the compressive stress, the higher the contact points, the lower the resistivity. In the present paper rheological properties, electrical resistivity and pressure-sensitive behavior under cyclic compressive loading of cement composites containing multi-walled carbon nanotubeswere investigated.

\section{Materials}

The experimental program was carried out on cement pastes and mortars containing different amount of MWNTs. Rheological properties of cementitious mixtures were evaluated. Electrical resistivity on unloaded and loaded cement paste and mortar specimens was measured.

An Ordinary Portland Cement (CE I 52.5R - according to EN 197/1) was used.Multi-Wall CNTs provided by Shenzhen NANO Tech. Port. Co. Ltd. (China) with average diameters about 10-30 nm were added in cement pastes and mortars. Their properties are given in Table 1. For mortars, CEN Standard sand (ISO standard sand) was used.

Table 1. Properties of multi-walled carbon nanotubes (CNTs)

\begin{tabular}{|c|c|c|c|}
\hline External diameter $[\mathrm{nm}]$ & $10 \div 30$ & Ash [\%] & $<0.2$ \\
\hline Length $[\mu \mathrm{m}]$ & $5 \div 15$ & Specific surface area $\left[\mathrm{m}^{2} / \mathrm{g}\right]$ & $70 \div 90$ \\
\hline Purity [\%] & $>95.0$ & Density $[\mathrm{ml} / \mathrm{g}]$ & $4 \div 6$ \\
\hline Amorphous carbon [\%] & $<2.0$ & Electric Conductivity $[\mathrm{S} / \mathrm{cm}]$ & $100 \div 0.0001$ \\
\hline
\end{tabular}

\section{Mixing procedure}

In order to form a conductive network and investigate their rheological and electrical properties, CNTs need to be fully dispersed in the cement matrix. In fact, CNTs tend to aggregate together and form nanotube clusters and bundles. In this research, two different methods to disperse CNTs for the fabrication of CNT/cement compositeswere used.

Method \#1. Carbon nanotubes were weighed and mixed with about $50 \%$ of mixing water in a beaker by means of a glass wand. Then, the solution was sonicated by an ultrasonic generator (Sonica ultrasonic cleaner) for 10 minutes. The remaining water and the cement (and the sand for the mortars) was finally added into the bowl and hand mixed for 3 minutes. Then, the bowl was positioned into the mixer. The mixing procedure adopted was that according to UNI EN 196$1: 2005[5]$.

Method \#2. An alternative method of dispersing CNTs in cement matrices is to use a noncovalent surface modification for CNTs surface. With a non-covalent interaction, surfactants can be wrapped around the nanotubes, favoring a better dispersion of CNTs in the aqueous solution. In this research, the critical micelle concentration of $1.4 \cdot 10^{-2} \mathrm{~mol} / 1$ of Sodium Linear Alkylbenzene Sulfonate (LAS)was used. The surfactant was firstly mixed with water using a magnetism stirred for $10 \mathrm{~min}$. Then, MWNTs were added into the aqueous solution and sonicated with an ultrasonicator for 2 hoursto make a uniformly dispersed suspension. Then a mortar mixer was used to mix this suspension and the cement (and the sand for the mortar) for 3 minutes. Finally a defoamer, tributyl phosphate, $(0.5 \%$ by cement volume $)$ was added into the mixture and mixed for another 3 minutes [6]. 
Four cement pastes with the same w/c ratio $(0.60)$ were prepared:

1.Paste containing $0.1 \%$ by cement mass of CNTs (PM1_0.1) dispersed by method \#1;

2. Pastecontaining $0.1 \%$ by cement mass of CNTs (PM2_0.1) dispersed by method \#2;

3. Pastecontaining $1.0 \%$ by cement mass of CNTs (PM1_1.0) dispersed by method \#1;

4. Paste containing $1.0 \%$ by cement mass of CNTs (PM2_1.0) dispersed by method \#2.

Four cementitiousmortars with the same w/c ratio $(0.60)$ were manufactured:

1.Mortar containing $0.1 \%$ by cement mass of CNTs (MM1_0.1) dispersed by method \#1;

2.Mortarcontaining $0.1 \%$ by cement mass of CNTs (MM2_0.1) dispersed by method \#2;

3.Mortarcontaining $0.5 \%$ by cement mass of CNTs (MM1_0.5) dispersed by method \#1;

4.Mortarcontaining $0.5 \%$ by cement mass of CNTs (MM2_0.5) dispersed by method \#2.

The specific mass of the fresh pastes and mortars was determined in order to evaluate the tendency of the mixture to entrap air.

\section{Preparation of cement composites specimens}

Fresh cement compositeswere poured into steel moulds immediately after the mixing. The mixtures was placed into the moulds $(40 \times 40 \times 160 \mathrm{~mm})$ in three layers and compacted by several jolts. Afterward, the composite in excess was eliminated by means of a scraper. Four copper electrodes $(50 \times 15 \times 2 \mathrm{~mm})$ were embedded into the fresh cementitiousmixtures to prepare four-probe conductance testing specimens (Figure 1). The two inner probes were positioned symmetrically at a distance of $40 \mathrm{~mm}$, while the outer at $60 \mathrm{~mm}$. Since the electrical resistivity measurements require the electrodes must be positioned in a precise way inside the specimens, a steel template was used $[4]$.
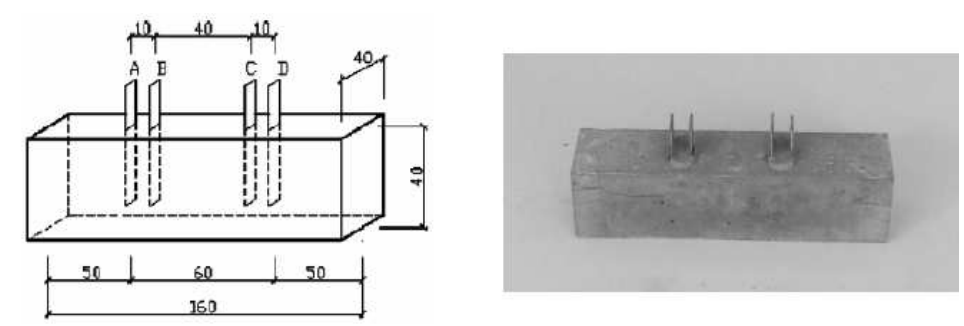

Figure 1 - Electrodes configuration in the specimen (size in $\mathrm{mm}$ )

\section{Curing conditions}

Moulds were stored in a room at $20 \pm 2^{\circ} \mathrm{C}$. All the specimens were demoulded the day after the mixing, then marked, soaked into water at $(20.0 \pm 1.0){ }^{\circ} \mathrm{C}$ for 7 days and finallydried for 24 hours in oven at $40 \pm 2^{\circ} \mathrm{C}$.

\section{Experimental tests}

The electrical resistance was measured by the four-probe method with a digital multimeter (Keithley, model 2001) on the unloaded specimen. Then the specimen was vertically positioned under the test machine and the electrodes were connected to the clamps of a multimeter. The resistance was measured increasing load, gradually applied through a force rate fixed at $0.02 \mathrm{KN} / \mathrm{sec}$. Three different load cycles were used to test the electrical responses of the specimens:

1. Seven load/unload cycles up to $12 \%$ of the compressive strength of the cementitious material;

2. load/unload cycles in theelastic range ( $4 \%, 8 \%, 12 \%$ of compressive strength);

3. load/unload cycles in theinelastic range (12\%, 20\%, 30\% of compressive strength). 


\section{Results and discussion}

Figure 2shows specific mass of cement pastes and mortars. The mixtures prepared usingmethod \#2 have a specific mass slightly lower (1.0\% and $5.0 \%$ for pastes and mortars, respectively) than that of composites manufactured with method \#1. The decrease in the specific mass of composite produced with method \#2 should be ascribed to the defoamer not able to remove completely the air entrapped by the surfactant.

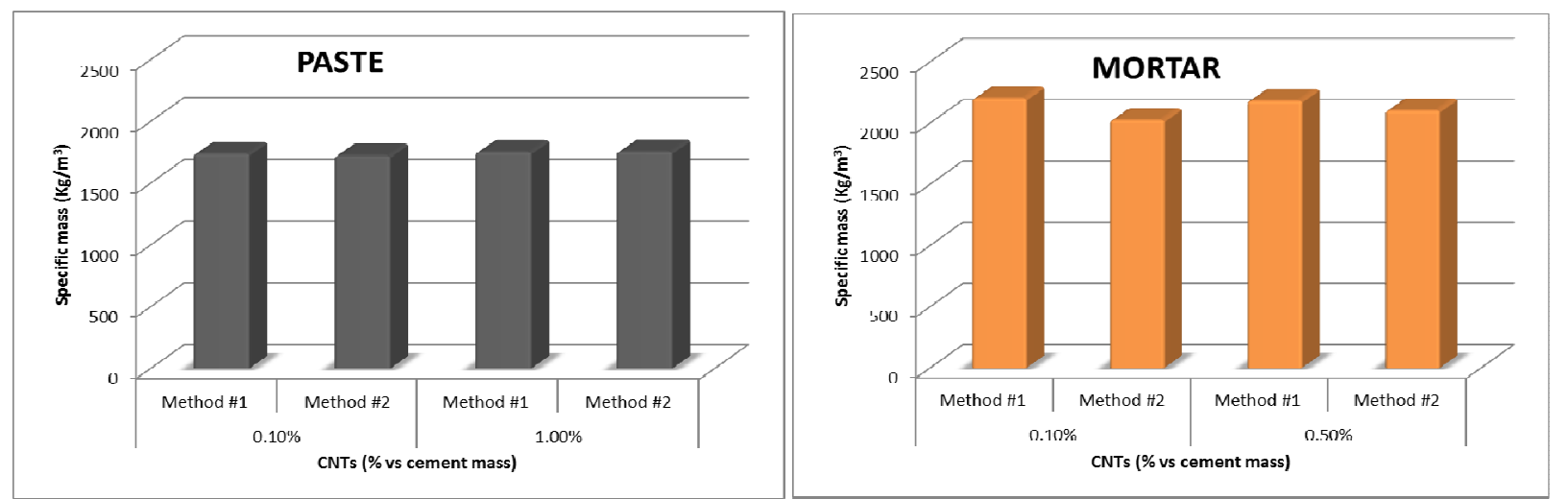

Figure 2-Specific mass of cement composites (cement pastes on the left; mortars on the right) as a function of the dispersion method and carbon nanotubes percentage.

Figure 3concerns electrical resistance values of cement pastes in unloaded condition for composites manufactured with both dispersion method \#1 and \#2 at different CNTs percentages. The addition of $0.1 \%$ of carbon nanotubes dispersed by method \#2 produces a markeddecrease in the electrical resistance. This means that method \#2 permits to obtain a better dispersion of carbon nanotubes in the cement matrix. The electrical resistance of cement pastes containing $1.0 \%$ of CNTs is substantially the same for the composites manufactured with the two dispersing method. Hence, data seem to indicate that this percentage $(1.0 \%)$ of CNTs exceeds the percolation threshold value. In other words, the number of nanotubes in the cement matrix is enough to form a continuous network independently of the dispersion method.

No significantly differences were detected in the electrical resistance between the mortars manufactured with the two CNTs dispersing methods (Figure 4). These results seem to indicate that the percentages $(0.1 \%$ and $0.5 \%$ vs. cement mass $)$ of CNTs used are too low to guarantee a conductive pattern even when the more effective dispersing method \#2 was adopted.

Figure 5 presents the variation of the electrical resistance of CNTs reinforced cement pastes under repeated compressive stress (12\% of the compressive strength). The electrical resistance remarkably changed for the cement pastes containing $0.1 \%$ of CNTs dispersed by method\#2. On the contrary, negligible changes in the electrical properties were noticed for cement pastes manufactured with method \#1, independently of the percentages of CNTs embedded in the cement matrix. Results highlight that an evident pressure sensitivity is achievable by means of an efficient dispersion (by using method \#2) of $0.1 \%$ CNTs. Increasing the dosage of CNTs up to $1.0 \%$ the stress sensitivity behavior is less evident because the difference in the number of contact points between the unloaded and loaded condition is lower. As stated in the percolation theory, the magnitude of the electrical conductivity increases with the CNTs content. However, independently of the stress level applied, a critical percentage exists and above this value further fibers addition will not produce any significantly variation in conductivity[7].

Figure 6 shows the change of the electrical resistance of CNTs reinforced cement pastes when compressive stress varies from $12 \%$ up to $30 \%$ of the compressive strength. A remarkably decrease of the electrical resistance was detected for the cement paste containing $0.1 \%$ of CNTs dispersed by method \#2. A lower change in the electrical resistivity was measured for the cement paste with the same percentage of CNTs added by using method \#1. Moreover, independently of the CNTs dispersion method, the higher the stress applied the lower the electrical resistance of the composite 
materials. Cement pastes with $1 \%$ of CNTs added by means of method 2 show a lower change in the electrical resistivity with respect variations of the same properties measured for the paste with $0.1 \%$ manufactured with the same dispersion method. Finally, a negligible reduction in the electrical resistance was achieved for the cement paste with $0.1 \%$ of CNTs dispersed by method \#1.

Data seem to indicate excellent piezoresistive properties when CNTs are added to the cement matrix by means of the more effective dispersion method $\# 2$, independently of the percentage of CNTs used, even if the lower dosage $(0.1 \%)$ is more sensitive to the variation of the stress applied. When method \#1 is used, since dispersion is not so efficient as method \#2, the piezoresistive properties are guaranteed only by using a higher dosage $(1.0 \%)$ of CNTs.

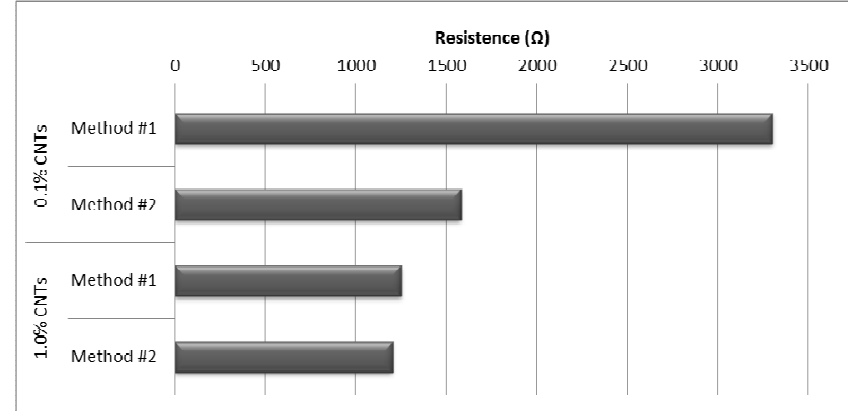

Figure 3-Electrical resistance of cement pastesin unloaded condition

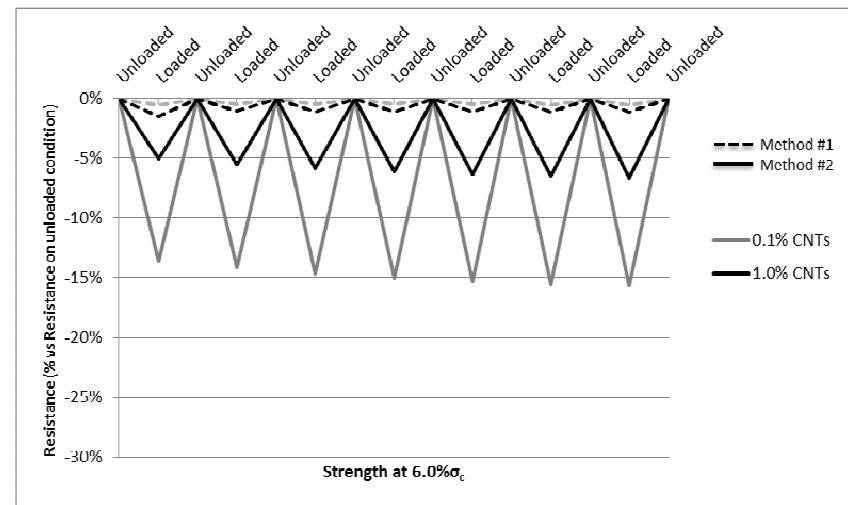

Figure 5- Cement paste electrical resistance change under load cyclesup to $12 \%$ of the compressive strength

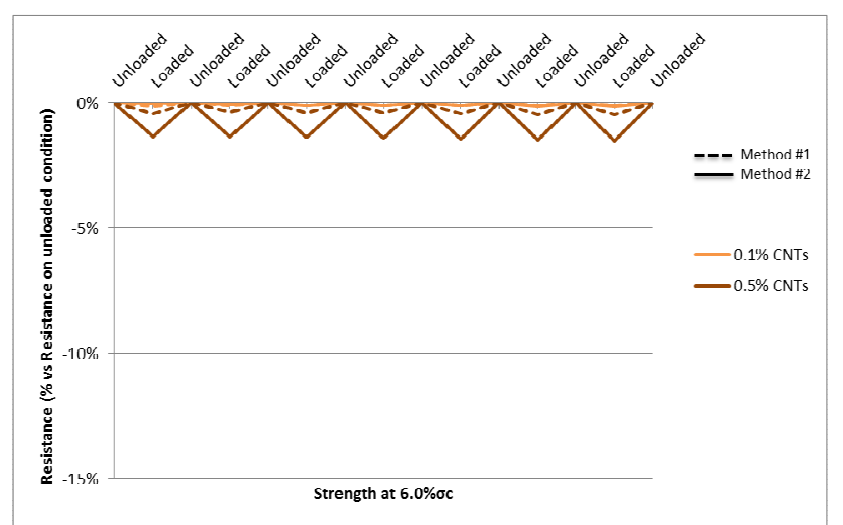

Figure 7- Mortar electrical resistance change under load cycles up to $12 \%$ of the compressive strength

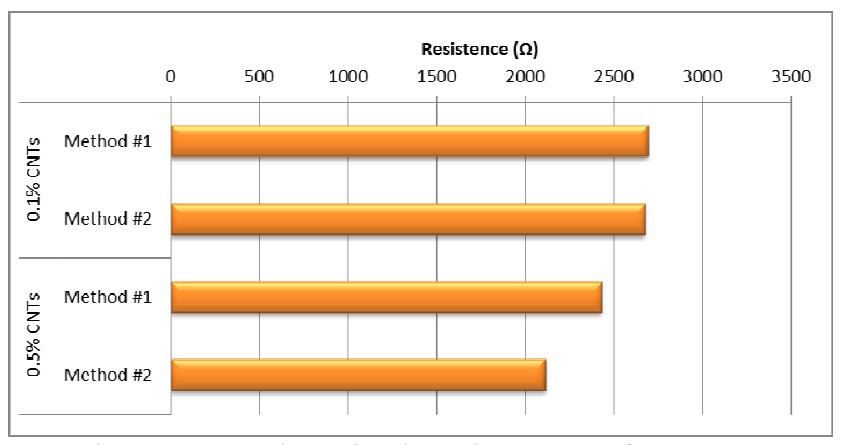

Figure 4 - Electrical resistance of cement mortarsin unloaded condition.

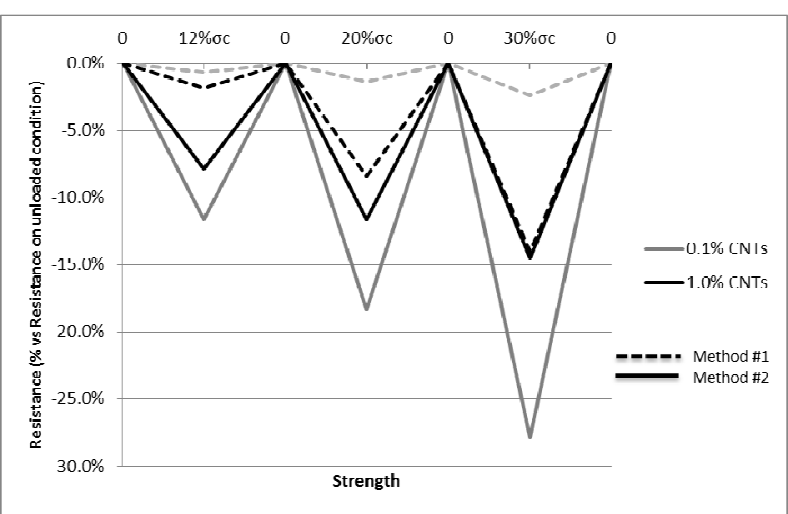

Figure 6-Cement paste electrical resistance change under inelasticload cycles up to $30 \%$ of the compressive strength

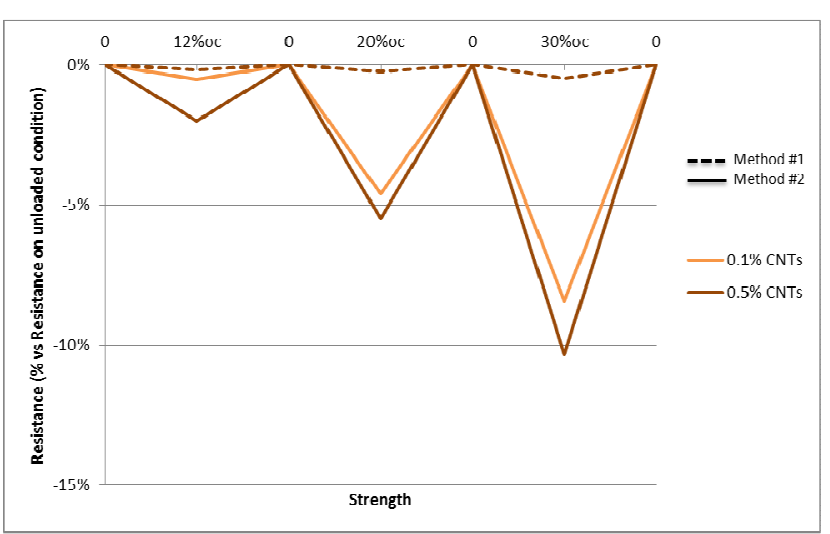

Figure 8-Mortar electrical resistance change under inelastic load cycles up to $30 \%$ of the compressive strength 
Figure 7 shows the variation of the electrical resistance of CNTs reinforced mortars. The electrical resistance changed in a negligible manner under repeated compressive loading. Data indicate that mortars are less pressure-sensible with respect cement pastes. Probably the CNTs percentage was too low to create a carbon nanotubes network inside the specimens when the stress applied was equal to $12 \%$ of the compressive strength. Figure 8 shows the change of the electrical resistance of mortarsby increasing compressive stress up to $30 \%$ of the compressive strength. Significant variations of electrical resistance were noticed only for mortars produced by dispersion method \#2.

\section{Conclusion}

Electrical resistivity of cement composites containing carbon nanotubes were investigated as a function of the percentage of CNTs, the dispersion method of CNTs in the cement matrix and the level of compressive stress acting on the cementitious material. In the absence of load the addition of $0.1 \%$ of carbon nanotubes dispersed by using a surfactant (method \#2) produces a marked decrease in the electrical resistance. Method \#2 permits to obtain a better dispersion of carbon nanotubes in the cement matrix with respect method \#1 based on a mere sonication of the aqueous solution containing CNTs. The electrical resistance of cement pastes containing $1.0 \%$ of CNTs is substantially the same for the composites manufactured with the two dispersing method confirming that this percentage $(1.0 \%)$ of CNTs exceeds the percolation threshold value. No significant differences were detected in the electrical resistance between the unloaded mortars manufactured with the two CNTs dispersing methods confirming that the percentages $(0.1 \%$ and $0.5 \%)$ of CNTs used are too low to guarantee a conductive pattern even when the more effective dispersing method \#2 was adopted.Excellent piezoresistive properties were achieved when CNTs are added to the cement matrix by means of the more effective dispersion method \#2, independently of the percentage of CNTs used, even if the lower dosage $(0.1 \%)$ is more sensitive to the variation of the stress applied. When method\#1 is used, since dispersion is not so efficient as method \#2 the piezoresistive properties are guaranteed only by using a higher dosage (1.0\%) of CNTs.

Data indicate that mortars are less pressure-sensible with respect cement pastes. Probably the CNTs percentages used were too low to create a carbon nanotubes network inside the specimens when the stress applied was equal to $12 \%$ of the compressive strength. When the compressive stress is as high as $30 \%$ of the compressive strength, significant variations of electrical resistance were noticed only for mortars produced by dispersion method \#2.

Based on the results of the present experimental research CNTs cement composites have great potential and they could be used in the next future in concrete field for practical applications to monitor the stress level of reinforced concrete elements subjected to static, dynamic and impact loads. In particular, informations on actual stress existing under dynamic and impact loads could be improve design procedures in protective structures.

\section{References}

[1] S. Iijima: Nature Vol. 354 (1991), pp.56-58;

[2] Wagner, H.D. et al., "Stress-induced fragmentation of multi-walled carbon nanotubes in a polymer matrix", ApplPhysLett, 1998; 72(2):188-90.

[3] G. Y. Li, P. M. Wang, X. Zhao: Carbon 43 (2005), pp. 1239-1245.

[4] G. Y. Li, P. M. Wang, X. Zhao: Cement\&Concrete Composites 29 (2007), pp.377-382.

[5] L. Coppola, E. Borgarello, A. Buoso, F. Corazza, E. Crippa, Proceedings of the 1st Workshop "Le nuove frontiere del calcestruzzo strutturale - The new boundaries of structural concrete", ACI ItalyChapter (Salerno, 2010), pp. 299-306;

[6] X. Yu,E. Kwon, Smart Mater. Struct. 18 (2009) 055010 (5pp)

[7] M. Chiarello, R. Zinno,Cement\& Concrete Composites 27 (2005) 463-469; 
Performance, Protection and Strengthening of Structures under Extreme Loading doi:10.4028/www.scientific.net/AMM.82

Electrical Properties of Carbon Nanotubes Cement Composites for Monitoring Stress Conditions in Concrete Structures

doi:10.4028/www.scientific.net/AMM.82.118 